\title{
Phase behavior of pure lipid bilayers with mismatch interactions
}

\author{
Zhang, Zhengping; Laradji, Mohamed; Guo, Hong; Mouritsen, Ole G.; Zuckermann, Martin
}

Published in:

Physical Review A

Link to article, DOI:

10.1103/PhysRevA.45.7560

Publication date:

1992

Document Version

Publisher's PDF, also known as Version of record

Link back to DTU Orbit

Citation $(A P A)$ :

Zhang, Z., Laradji, M., Guo, H., Mouritsen, O. G., \& Zuckermann, M. (1992). Phase behavior of pure lipid bilayers with mismatch interactions. Physical Review A, 45(10), 7560-7567.

https://doi.org/10.1103/PhysRevA.45.7560

\section{General rights}

Copyright and moral rights for the publications made accessible in the public portal are retained by the authors and/or other copyright owners and it is a condition of accessing publications that users recognise and abide by the legal requirements associated with these rights.

- Users may download and print one copy of any publication from the public portal for the purpose of private study or research.

- You may not further distribute the material or use it for any profit-making activity or commercial gain

- You may freely distribute the URL identifying the publication in the public portal 


\title{
Phase behavior of pure lipid bilayers with mismatch interactions
}

\author{
Zhengping Zhang, Mohamed Laradji, and Hong Guo \\ Centre for the Physics of Materials, Department of Physics, McGill University, Rutherford Building, \\ 3600 University Street, Montréal, Québec, Canada H3A 2 T8 \\ O.G. Mouritsen and M.J. Zuckermann \\ Department of Physical Chemistry, The Technical University of Denmark, DK-2800 Lyngby, Denmark
}

(Received 14 October 1991)

\begin{abstract}
Recently Corvera, Laradji, and Zuckermann (unpublished) showed that the multistate lattice model due to Pink, Green, and Chapman [Biochemistry 20, 6692 (1981)] with parameters obtained from fitting to thermodynamic data for saturated phospholipid bilayers does not exhibit a phase transition but could instead be described in terms of short-range fluctuations close to a critical point. We have extended the Pink-Green-Chapman model by including hydrophobic mismatch interactions between the lipid acyl-chain conformational states. We used Monte Carlo techniques to examine the phase behavior of the extended model and found that it exhibits first-order phase transitions above a critical value of the mismatch parameter. The results are discussed in relation to previous theoretical work as well as experimental measurements on lipid bilayers.

PACS number(s): $87.22 . \mathrm{Bt}, 64.60 . \mathrm{Cn}$
\end{abstract}

\section{INTRODUCTION}

Fully hydrated lipid bilayers have been the subject of great interest both for their intriguing thermodynamic properties and as models of biological membranes [1]. One field of considerable endeavor involves the experimental and theoretical study of the phase behavior of these systems, which show considerable polymorphism. Here we concentrate on a theoretical description of the main phase transition of pure lipid bilayers in which the bilayer passes from a gel (solid) to a liquid-crystalline (fluid) phase. Experimentally, this transition is characterized by a sharp peak in the specific heat and dramatic changes in acyl-chain conformational order parameters. Despite the appearance of very strong fluctuations at the main transition, this transition is usually regarded as a first-order phase transition and this is reflected in the results found by several theoretical models. However, the interpretation of the experimental data in terms of a first-order transition is by no means ambiguous [2] and the true nature of the main transition is still in dispute. It is hence of interest to analyze theoretical, microscopic interaction models of the bilayer phase behavior in order to shed some light on which couplings control the nature of the transition and its appearance.

In the present paper we study by computer simulation the nature of the lipid bilayer main transition within a microscopic interaction model, taking full advantage of a recently proposed method of Lee and Kosterlitz [3]. This method is very powerful in assessing the order of phase transitions. The model belongs to a class of statisticalmechanical models for the bilayer transition $[4,5]$ that take due account of the strong acyl-chain lateral density fluctuations. These fluctuations dominate the transition region, and it is therefore rather difficult, using conventional methods of analyzing the transition [5], to assess whether the transition is continuous or of first order, or whether there is a transition at all. The model we study is related to the Pink-Green-Chapman (PGC) multistate lattice model [4], which was one of the first two-dimensional lattice models used to examine closely the nature of the main phase transition. The PGC model was first examined in the mean-field approximation [4] and the model parameters used were determined by fitting to thermodynamic data for saturated phosphatidylcholine (PC) or lecithin bilayers of different acyl-chain length. The mean-field calculations predicted sharp firstorder phase transitions for all chain lengths (12-22) examined. Mouritsen and co-workers [5] reported the results of Monte Carlo calculations that showed that the thermodynamic properties and response functions of the PGC model had an abrupt but continuous behavior in the transition region. Furthermore, these results showed that the model exhibited dynamic heterogeneity in the transition region, which could be interpreted in terms of thermal fluctuations in the form of clusters of the minority phase in the majority phase. Ipsen, Jørgensen, and Mouritsen [6] suggested that the fluctuations could be characterized by a finite length scale and that they behaved in a pseudocritical manner in the transition region. They concluded that, for the fitted parameters used in the mean-field approximation, the main transition was a first-order phase transition but extremely close to a critical point. They also found that the response functions (specific heat and lateral compressibility) sharpened with increasing chain length. In contrast, Corvera, Laradji, and Zuckermann [7], using the method of Lee and Kosterlitz [3], found no phase transition in the PGC model for the fitted parameters. They showed that the system was close to a critical point and that the dynamic heterogeneity [5] predicted by the PGC model is related to long-lived short-range order effects. Corvera, 
Laradji, and Zuckermann [7] also found that a phase transition was absent for chain lengths up to 18 carbon atoms and that the first-order transition could be recovered by lowering the effective surface pressure, which represents polar-head interactions in the PGC model. Their conclusions are confirmed by Ipsen [8], who found that the finite-size behavior of the peak in the specific heat remained constant with varying system size up to lattices of size $300 \times 300$; i.e., it does not scale as expected for a first-order phase transition.

The above calculations [4-8] were performed for a single monolayer of the bilayer and the question therefore arises: What type of interactions present in bilayers and not in monolayers leads to first-order phase transitions? One type of interaction is a mismatch interaction between lipids in different conformational states, which is analogous to the interaction of the "mattress model" proposed by Mouritsen and Bloom [9] for lipid-protein interactions in bilayers. In their model an important interaction was assigned to the mismatch between neighboring lipids and proteins with unequal interfacial lengths of hydrophobic contact. This effect was represented by a repulsive interaction between the aqueous medium and the superfluous hydrophobic length. We argue that the same interaction occurs between neighboring lipids in different rotameric conformational states having unequal hydrophobic contact lengths. This interaction only exists in bilayers, since, for monolayers, the superfluous hydrophobic length can make contact with the air (or oil) at negligible energy cost.

In this paper we report numerical simulations using the Metropolis-Monte Carlo method for the PGC model extended to include the lipid-chain mismatch interaction as described in Sec. II. We show that this model exhibits a well-defined first-order phase transition for a sufficiently large value of the mismatch interaction. We examine the nature of the transition as a function of the strength of the mismatch interaction and give an approximate value for the critical point. The methods used to identify the nature of the phase behavior from Monte Carlo data are due to Binder [10], Ferrenberg and Swendsen [11], and Lee and Kosterlitz $[3,12]$. These methods are described in Sec. III. The results of the simulation are given in Sec. IV, and Sec. V concludes the paper with a discussion.

\section{MICROSCOPIC MODEL}

The principal term in the Hamiltonian of our extended model is the multistate lattice model due to Pink, Green, and Chapman [4]. The PGC model has been used to examine the phase behavior and dynamics of pure phospholipid bilayers using both the mean-field approximation and numerical simulations based on the MetropolisMonte Carlo method, as mentioned in the Introduction. The PGC model takes into account the acyl-chain conformational statistics and the van der Waals interactions among various conformers in a detailed manner, while the excluded-volume effect is partially accounted for by assigning each lipid chain to a single site of a triangular lattice. The acyl-chain conformations are represented by ten single-chain states, $\alpha=1, \ldots, 10$, each described by a cross-sectional area $A_{\alpha}$, an internal energy $E_{\alpha}$, and an internal degeneracy $D_{\alpha}$. The nine lower states are characteristic of the gel phase and the tenth state is typical of the fluid phase. Of the nine gel states, the lowest state is the all-trans ground state and the eight remaining states are low-lying conformational excitations of the ground state. The Hamiltonian of the PGC model can be written as follows:

$$
\begin{aligned}
\mathcal{H}_{0}= & \sum_{i} \sum_{\alpha=1}^{10}\left(E_{\alpha}+\Pi A_{\alpha}\right) \mathcal{L}_{\alpha, i} \\
& -\frac{J_{0}}{2} \sum_{\langle i, j\rangle} \sum_{\alpha, \beta=1}^{10} I_{\alpha} I_{\beta} \mathcal{L}_{\alpha, i} \mathcal{L}_{\beta, j},
\end{aligned}
$$

where $\langle i, j\rangle$ are nearest-neighbor indices. $J_{0}$ is the strength of the van der Waals interaction, which was determined by a fit to experimental values of the transition temperature $T_{\mathrm{m}}$ and the transition enthalpy $\Delta \mathcal{H} . \mathcal{L}_{\alpha, i}$ is an occupation variable, which is unity when the $i$ th chain is in the $\alpha$ th conformational state and zero otherwise. $I_{\alpha}$ is a product of a term related to the van der Waals interaction among chains and a phenomenological expression for the shape-dependent nematic parameter due to Seelig and Seelig [13]. II represents the effective lateral pressure exerted on a lipid bilayer due to interfacial effects (hydration, polar head interactions, etc.). The parameters $J_{0}$ and $\Pi$ were found by fitting to experimental values for the transition temperatures and transition enthalpies of pure saturated PC bilayers [4]. The values found for dipalmitoyl phosphatidylcholine (DPPC) are $J_{0} \approx 0.71 \times 10^{-13}$ erg and $\Pi=30$ dyn $/ \mathrm{cm}$.

An additional term in the Hamiltonian describes the mismatch interaction among different conformational states of the acyl chains and can be written as follows by analogy with the case of lipid-protein interactions [9, 14]:

$$
\mathcal{H}_{\mathrm{mis}}=\frac{\gamma_{\mathrm{mis}}}{2} \sum_{\langle i, j\rangle} \sum_{\alpha, \beta}\left|d_{\alpha}-d_{\beta}\right| \mathcal{L}_{\alpha, i} \mathcal{L}_{\beta, j},
$$

where $d_{\alpha}$ is the hydrocarbon chain length for the $\alpha$ th conformational state, which is directly related to the value of $A_{\alpha}$ since the volume of an acyl chain is assumed invariant under temperature changes. The parameter $\gamma_{\text {mis }}$ is related to the hydrophobic effect. The total Hamiltonian of the extended model is therefore

$$
\mathcal{H}=\mathcal{H}_{0}+\mathcal{H}_{\text {mis }}
$$

Results of numerical simulations based on this model and using the methods described in the following section are reported in Sec. IV.

\section{CALCULATIONAL TECHNIQUES}

First-order transitions are characterized by discontinuities in the first derivatives of the free energy. This results in the thermodynamic limit in a $\delta$-function singularity for the specific heat $C(T)$ and a singularity for the compressibility $\chi(T)$ at the transition. In a finite system, however, the transition region is broadened, the peaks in 
$C(T)$ and $\chi(T)$ are finite, and their height increases with increasing linear lattice size $L$. Furthermore, the location of the intensity of the maxima varies in a size-dependent manner. The maxima grow as $L^{d}$ in $d$ dimensions and the $\delta$-function limit is obtained because the width decreases as $L^{-d}$. The maximum value of $C(T)$ for a finite two-dimensional $(d=2)$ lattice is

$$
C_{L}^{\max }=a+b L^{d}
$$

where $a$ and $b$ are the size-independent parameters, which are model dependent [15]. A similar scaling form for the maximum value of the compressibility $\chi_{L}^{\max }$ also exists. $C(T)$ and $\chi(T)$ are calculated from the fluctuationdissipation theorem

$$
\begin{aligned}
& \chi(T)=\beta\left(\left\langle A^{2}\right\rangle-\langle A\rangle^{2}\right) / L^{d}, \\
& C(T) / k_{B}=\beta^{2}\left(\left\langle\mathcal{H}^{2}\right\rangle-\langle\mathcal{H}\rangle^{2}\right) / L^{d},
\end{aligned}
$$

where $\beta=1 / k_{B} T$ and

$$
A=\sum_{\alpha, i} A_{\alpha} \mathcal{L}_{\alpha, i}
$$

The phase behavior of the PGC model in Eq. (1) was previously examined by the standard Metropolis-Monte Carlo method with importance sampling and "spin-flip" dynamics [5]. This method is used here in conjunction with the new techniques of Ferrenberg and Swendsen [11] and Lee and Kosterlitz $[3,12]$ to examine the nature of the phase transition for the extended model in Eqs. (1)-(3). The Monte Carlo techniques used here consist of three parts. First, Monte Carlo simulations are performed for the evaluation of the specific heat $C(T)$ and the lateral compressibility $\chi(T)$ as functions of temperature $T$, using Eqs. (5)-(7). The results of these simulations are therefore used to determine the peaks in $C(T)$ and $\chi(T)$ as accurately as possible.

Extensive simulations are then performed at the position of the peak of $C(T)$ for several values of the system size $L$. The extrapolation method of Ferrenberg and Swendsen [11] is then used to calculate the probability distribution $P(E, A, T, L)$ of the internal energy and the area per lipid chain at the peak. The method of Ferrenberg and Swendsen uses standard thermodynamics to derive the probability distribution at neighboring values of the temperature. This allows us to calculate the values of $C(T)$ and $\chi(T)$ at neighboring temperatures. Finite-size scaling can then be performed on the data. For a firstorder phase transition, $C(T)$ scales according to Eq. (4), whereas it scales as $L^{\alpha / \nu}$ for a continuous transition, $\alpha$ and $\nu$ being the critical exponents characterizing the singularities af the specific-heat and the correlation length, respectively.

The order of the phase transition was then examined by the method of Lee and Kosterlitz [3, 12]. This method consists of calculating the free energy as a function of the order parameter at the transition from the probability distribution at the specific-heat peak. In our case we use the average area per lipid chain as the order parameter since this is coupled to the internal lateral pressure $\Pi$, $\mathrm{cf}$. Eq. (1). We therefore need to determine the probability distribution of the cross-sectional areas of the acyl chains, $\mathcal{P}(A, T, L)$, given by

$$
\mathcal{P}(A, T, L)=\sum_{E} \mathcal{P}(E, A, T, L) .
$$

The free-energy-like quantity $\mathcal{A}(A, T, L)$ defined by [12]

$$
\mathcal{A}(A, T, L) \sim-\ln \mathcal{P}(A, T, L)
$$

differs from the bulk free energy $\mathcal{F}(A, L)$ by a temperature- and $L$-dependent additive quantity. However, at fixed $T$ and $L$, the shape of $\mathcal{A}(A, T, L)$ will be identical to that of $\mathcal{F}(A, L)$ and furthermore $\mathcal{A}(A)-$ $\mathcal{A}\left(A^{\prime}\right)=\mathcal{F}(A)-\mathcal{F}\left(A^{\prime}\right)$. A measurement of $\Delta \mathcal{A}=$ $\mathcal{A}(A)-\mathcal{A}\left(A^{\prime}\right)$ therefore gives a direct evaluation of the corresponding $\Delta \mathcal{F}=\mathcal{F}(A)-\mathcal{F}\left(A^{\prime}\right)$. At a first-order transition, $\mathcal{F}(A, L)$ has pronounced double minima corresponding to two coexisting phases at $A=A_{1}$ and $A=A_{2}$ separated by a barrier with a maximum at $A_{\max }$ corresponding to a domain boundary between the two phases. The height of the barrier measures the interfacial free energy between the two coexisting phases and is given by

$$
\Delta \mathcal{F}(L)=\mathcal{F}\left(A_{\max }, L\right)-\mathcal{F}\left(A_{1}, L\right) \sim L^{d-1} .
$$

Therefore, at a first-order transition, $\Delta \mathcal{F}(L)$ increases monotonically with $L$.

The fourth-order cumulant $\sigma(L)$ is a useful quantity to measure in simulations, and behaves quite differently at first-order and continuous transitions [10]. For the energy $E$, this quantity is defined by

$$
\sigma(L)=1-\frac{\left\langle E^{4}\right\rangle}{3\left\langle E^{2}\right\rangle^{2}}
$$

For continuous transitions, $\sigma(L) \rightarrow \frac{2}{3}$ for all temperatures as $L \rightarrow \infty$. For first-order transitions, $\sigma(L)$ takes on the value $2 / 3$ for high and low temperatures, tending toward a nontrivial minimum value at the transition temperature. This minimum value of $\sigma(L), \sigma(L)_{\mathrm{min}}$, scales as $L^{-d}$ in the thermodynamic limit.

\section{RESULTS}

The approximation made in this paper is that the two monolayers of a phospholipid bilayer are identical and do not interact with one another. The two monolayers only react to each other indirectly via the mismatch interaction, Eq. (2). Therefore only one monolayer of the bilayer as described by the Hamiltonian of Eqs. (1) to (3) needs to be simulated. The simulation for a bilayer composed of two directly interacting monolayers will be presented in a later paper [16]. We examined this model by performing numerical simulations, using the methods described in Sec. III, on $L \times L$ triangular lattices with periodic boundary conditions. Very long simulations were performed at the transition temperature in order to obtain good statistics $[3,15]$. The temperatures at which the simulations were performed were chosen as close as possible to the transition temperature $T_{\mathrm{m}}(L)$, and it was found that $2 \times 10^{6}$ Monte Carlo steps per site (MCS) were sufficient to obtain the required accuracy. 

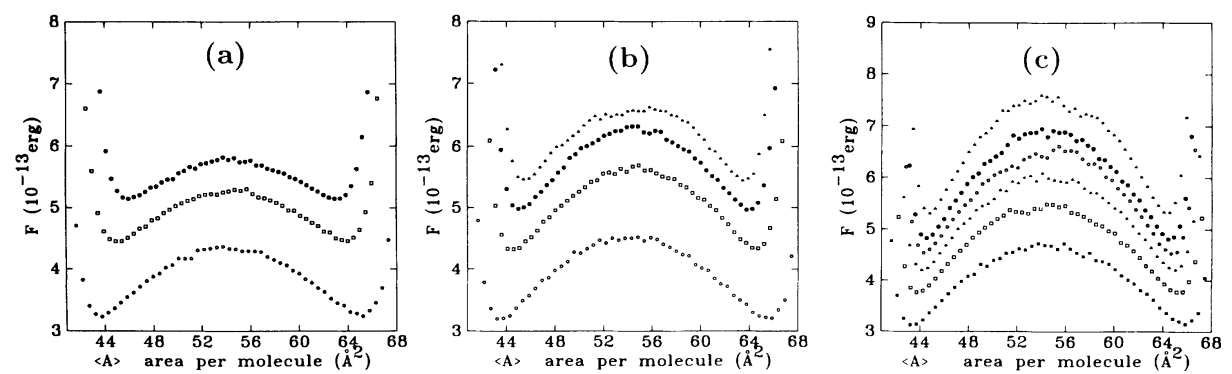

FIG. 1. Free energy $\mathcal{F}\left(T_{m}(L)\right)$ as a function of average molecular area $A$, shown for different values of the mismatch parameter $\gamma_{\mathrm{mis}}$ and for different linear lattice dimensions $L$. (a) $\gamma_{\mathrm{mis}}=3 \times 10^{-16} \mathrm{erg} / \AA$ and $L=8,16,24$ (from bottom to top). (b) $\gamma_{\mathrm{mis}}=4 \times 10^{-16} \mathrm{erg} / \AA$ and $L=8,16,24,32$ (from bottom to top). (c) $\gamma_{\mathrm{mis}}=5 \times 10^{-16} \mathrm{erg} / \AA$ and $L=8,12,16,20,24,32$ (from bottom to top).

The temperature at which a finite system undergoes a first-order phase transition usually depends on the size of the system. It also depends on which quantity is used to identify the finite-size transition point. In the case of the free energy, the transition temperature $T_{m}^{\mathcal{F}}(L)$ is defined by requiring $\mathcal{F}\left(A_{1}, L\right)=\mathcal{F}\left(A_{2}, L\right)$, where $\mathcal{F}\left(A_{1}, L\right)$ and $\mathcal{F}\left(A_{2}, L\right)$ are the free energies of the gel and fluid phases, respectively. The free energies were calculated for several values of the mismatch parameter $\gamma_{\mathrm{m} i \mathrm{~s}}$, in order to examine the phase behavior of the system and to locate the critical point. We found that the system did not exhibit a phase transition for values of $\gamma_{\text {mis }}$ below $4 \times 10^{-16} \mathrm{erg} / \AA$. At this value of $\gamma_{\text {mis }}$, the system is at or extremely close to the critical point. Above this value of $\gamma_{\text {mis }}$ the transition is of first order. The data on which these conclusions are based are presented in Fig. 1, which show that the free energy as a function of area per lipid chain exhibits two minima with a barrier between. The height of this barrier, $\Delta \mathcal{F}(L)$, changes with system size very differently for different values of $\gamma_{\mathrm{m} \text { s }}$. This can be seen in Fig. 2, where $\Delta \mathcal{F}(L)$ is shown as a function of system size for the three values of $\gamma_{\text {mis }}$ corresponding to Figs. 1(a)-1(c). Figure 2 shows that $\Delta \mathcal{F}(L)$ decreases

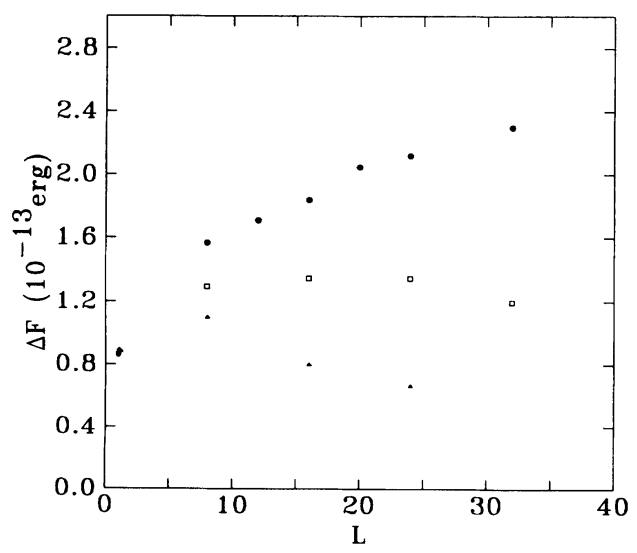

FIG. 2. Barrier height, $\Delta F\left(T_{m}(L)\right)$ in Eq. (10), for $\gamma_{\mathrm{mis}}=$ $3,4,5 \times 10^{-16} \mathrm{erg} / \AA$ (from bottom to top) as a function of linear lattice size $L$. with increasing $L$ for $\gamma_{\text {mis }}=3 \times 10^{-16} \mathrm{erg} / \AA$, implying the absence of a transition, and increases with increasing $L$ for $\gamma_{\text {mis }}=5 \times 10^{-16} \mathrm{erg} / \AA$, implying the occurrence of a first-order phase transition. For $\gamma_{\text {mis }}=4 \times 10^{-16}$ erg/ $\AA$, the height of the barrier does not depend on system size to within calculational error, indicating that the transition for this parameter value is very close to being a continuous transition. Figure 3 gives $\mathcal{F}(L)$ as a function of $\gamma_{\mathrm{mis}}$ for $\gamma_{\mathrm{mis}}=1,2,3,4,5 \times 10^{-16} \mathrm{erg} / \AA$ and $L=24$. This figure shows the emergence of the first-order phase transition with increasing $\gamma_{\mathrm{m} \text { s }}$ in terms of the appearance of the maximum in $\Delta \mathcal{F}(L)$.

Results for the fourth-order cumulant are given in Fig. 4 for $\gamma_{\text {mis }}=5 \times 10^{-16} \mathrm{erg} / \AA$. As $L$ increases, the minimum of the cumulant goes to a value different from $2 / 3$ at a certain temperature $T_{m}^{\sigma}(L)$, and to $2 / 3$ at other temperatures, as expected. The nontrivial limit is obtained by extrapolating the data for the larger sizes, as shown in Fig. 5.

The temperature dependence of the specific heat and the susceptibility as obtained using the method of Ferrenberg and Swendsen [11] are shown in Figs. 6 and 7, respectively, for $\gamma_{\text {mis }}=5 \times 10^{-16} \mathrm{erg} / \AA$ and for several sys-

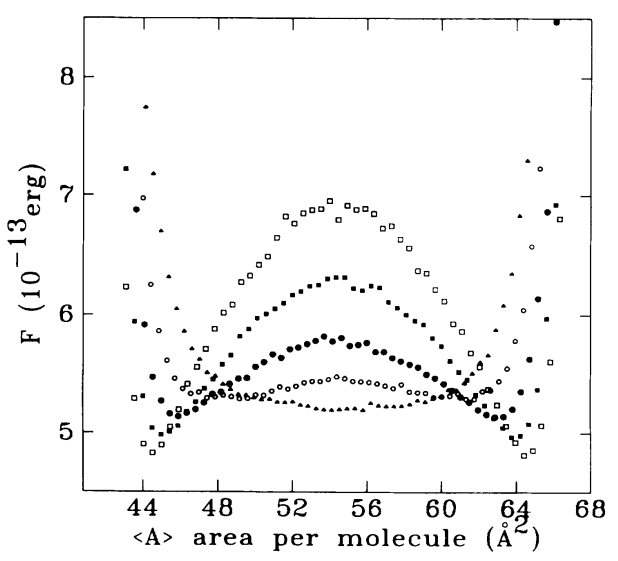

FIG. 3. Free energy $\mathcal{F}\left(T_{m}(L)\right)$ for $\gamma_{\text {mis }}=1,2,3,4,5 \times$ $10^{-16} \mathrm{erg} / \AA$ (from bottom to top) and $L=24$. 


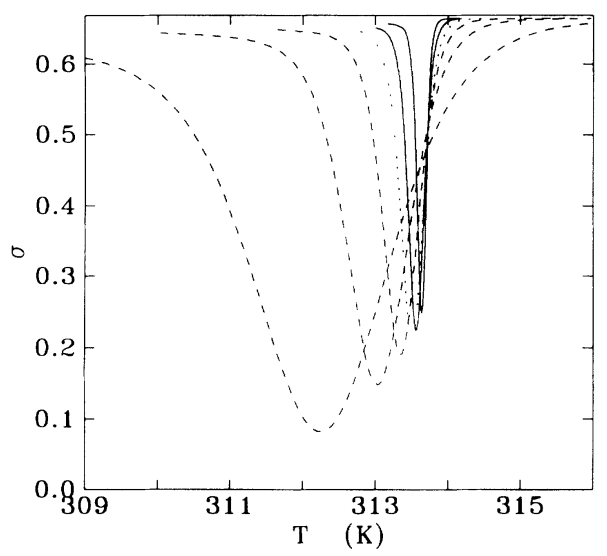

FIG. 4. Fourth-order cumulant, $\sigma(L)$ in Eq. (11), as a function of temperature for $L=8,12,16,20,24,32$ (from left to right) and $\gamma_{\mathrm{mis}}=5 \times 10^{-16} \mathrm{erg} / \AA$. The data were obtained from the same simulations as used for Fig. 1(c).

tem sizes. As the system size increases, the peak height of both response functions increases while the width of the peaks decreases. The peak heights of both quantities are plotted as functions of $L^{d}$ in Figs. 8 and 9 and a linear behavior is observed in agreement with Eq. (4). The full finite-size scaling behavior is investigated in Fig. 10 in the case of the specific heat. The expected scaling behavior for a first-order phase transition is clearly confirmed by this figure. The temperature $T_{m}^{C}$ at which the specific heat exhibits a maximum is, for large-enough system sizes, identical to the corresponding temperature for the susceptibility maximum. For finite system sizes, the values of $T_{m}^{\mathcal{F}}, T_{m}^{\sigma}$ and $T_{m}^{C}$ are, however, different, but they should tend to the same temperature in the thermodynamic limit, i.e., as $L \rightarrow \infty$. This is demonstrated in Fig. 11, which shows that for $\gamma_{\text {mis }}=5 \times 10^{-16} \mathrm{erg} / \AA$ the transition temperature is $T_{m}=313.7 \mathrm{~K}$ in the thermodynamic limit.

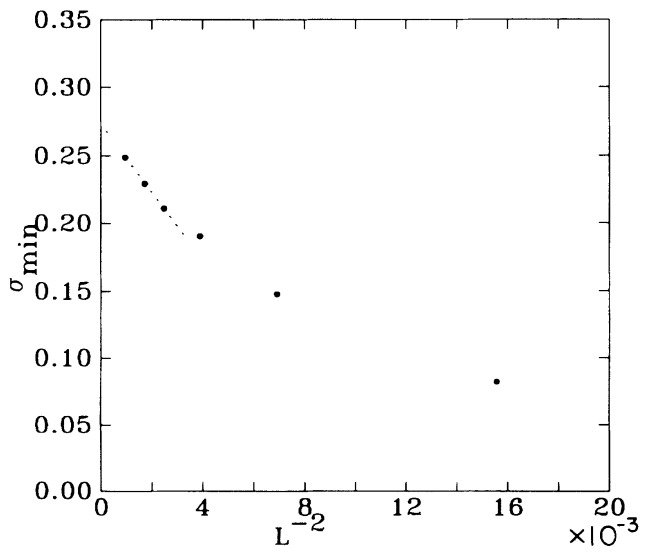

FIG. 5. Dependence of $\sigma(L)_{\min }$, cf. Fig. 4, on $L^{-d}$. Extrapolation to the non-trivial limit is indicated by the dashed line.

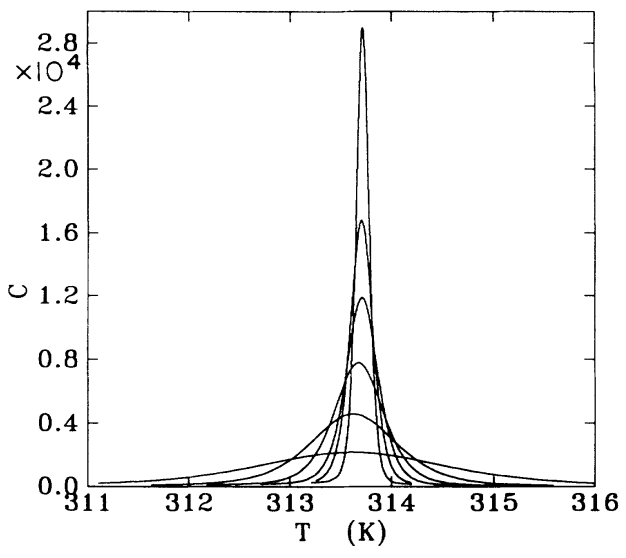

FIG. 6. Temperature dependence of the specific heat, $C(T)$, for $L=8,12,16,20,24,32$ and $\gamma_{\mathrm{mis}}=5 \times 10^{-16} \mathrm{erg} / \AA$. The specific heat is in units of $k_{B}$.

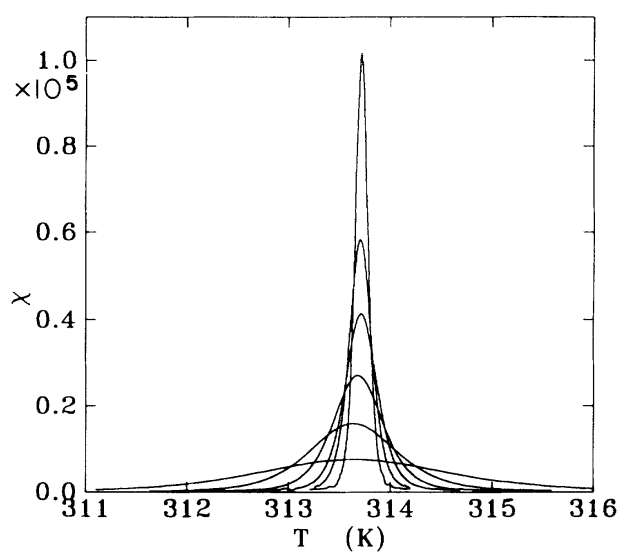

FIG. 7. Temperature dependence of the lateral compressibility, $\chi(T)$, for $L=8,12,16,20,24,32$ and $\gamma_{\mathrm{mis}}=$ $5 \times 10^{-16} \mathrm{erg} / \AA$. The lateral compressibility is in units of $10^{13} \AA^{4} \mathrm{erg}^{-1}$.

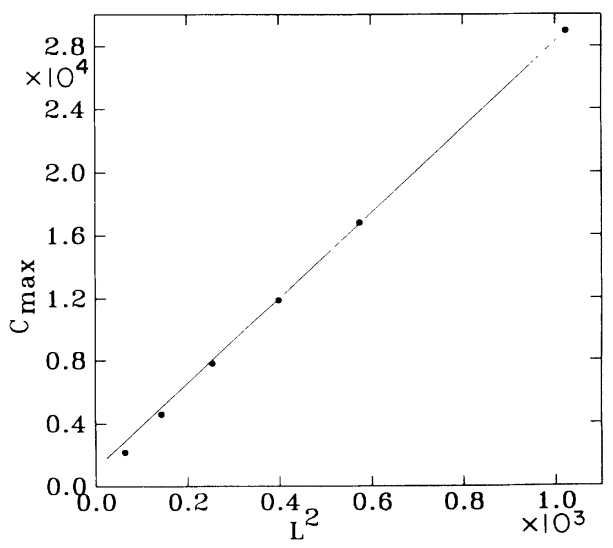

FIG. 8. Dependence of the specific-heat maximum on $L^{d}$ for $L=8,12,16,20,24,32$ and $\gamma_{\mathrm{mis}}=5 \times 10^{-16} \mathrm{erg} / \AA$. The specific heat is in units of $k_{B}$. 


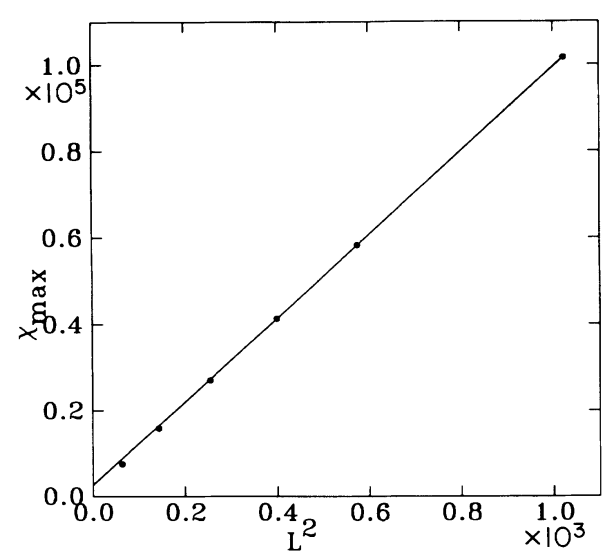

FIG. 9. Dependence of the lateral compressibility maximum on $L^{d}$ for $L=8,12,16,20,24,32$ and $\gamma_{\mathrm{mis}}=5 \times$ $10^{-16} \mathrm{erg} / \AA$. The lateral compressibility is in units of $10^{13} \AA^{4} \mathrm{erg}^{-1}$.

\section{DISCUSSION AND CONCLUSION}

We have in the present paper studied by computersimulation methods the nature of the phase transition in an extended version of the multistate PGC model [4] for the main transition in aqueous lipid bilayers. The study was motivated by the recent finding [7] of the absence of a phase transition in the standard PGC model for parameter values that are believed to represent DPPC bilayers [5]. The standard PGC model does not distinguish between a lipid monolayer and a bilayer. In the extended model, Eqs. (1)-(3), such a distinction is made by accounting for the hydrophobic incompatibility of neighboring acyl chains. The extended model is characterized by a so-called mismatch parameter $\gamma_{\text {mis }}$. We have shown, using a finite-size scaling analysis built on the Lee-Kosterlitz trick [3, 12], that the extended model displays a first-order phase transition above a critical value

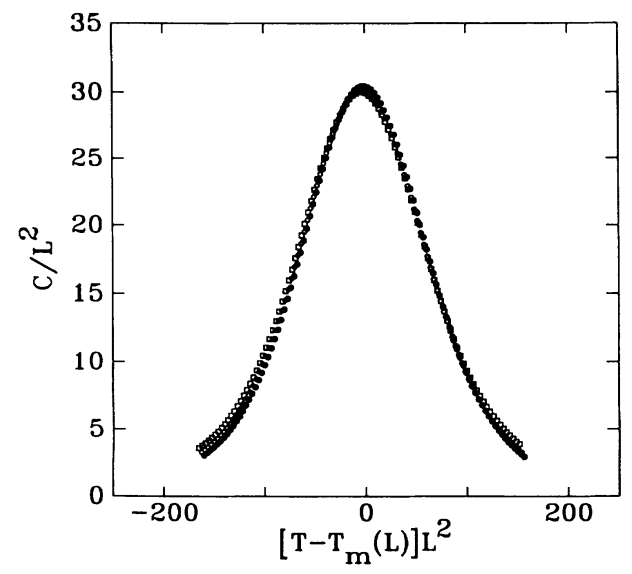

FIG. 10. Scaling function for the specific heat for $L=$ $8,12,16,20,24,32$ and $\gamma_{\mathrm{mis}}=5 \times 10^{-16} \mathrm{erg} / \AA$. The specific heat is in units of $k_{B}$.

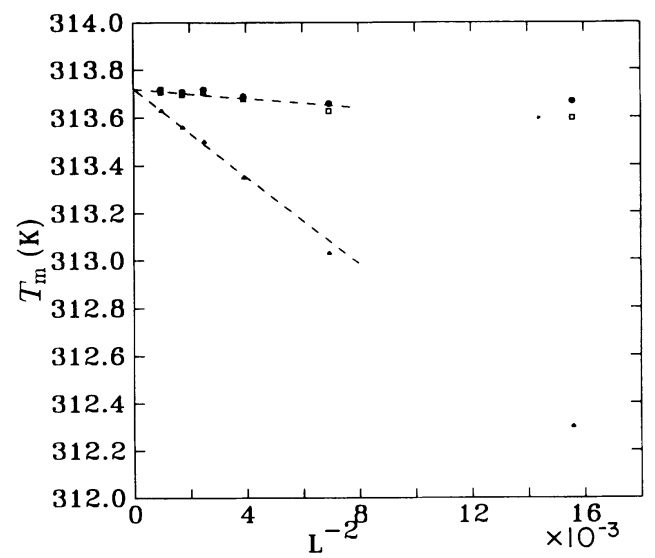

FIG. 11. Finite-size transition temperatures $T_{m}^{\mathcal{F}}(L)(\square)$, $T_{m}^{C}(L)(\bullet)$, and $T_{m}^{\sigma}(L)(\triangle)$, as determined by the free energy, $\mathcal{F}\left(A_{1}, T_{\mathrm{m}}(L)\right)=\mathcal{F}\left(A_{2}, T_{\mathrm{m}}(L)\right)$, the maximum of the specific heat (or lateral compressibility), and the minimum of the fourth-order cumulant, respectively. The mismatch parameter is $\gamma_{\mathrm{mis}}=5 \times 10^{-16} \mathrm{erg} / \AA$.

of the mismatch parameter. We examined this transition in detail for $\gamma_{\text {mis }}=5 \times 10^{-16} \mathrm{erg} / \AA$ and we showed that the transition temperature is $T_{\mathrm{m}}=313.7 \mathrm{~K}$ in the thermodynamic limit for this value of $\gamma_{\text {mis }}$. The absolute value of the mismatch interaction required to induce a first-order phase transition is small compared to the strength of van der Waals interactions among lipid chains. The ratio of the maximum value of the mismatch interaction used in this work to the coupling constant of the van de Waals interaction is only of the order of $5 \%$.

Based on experimental and theoretical data it has been suggested by a number of workers [17] that the main transition in lipid bilayers may be pseudocritical, i.e., very close to a critical point. The effect of the mismatch interaction is to suppress the fluctuations and drive the transi-

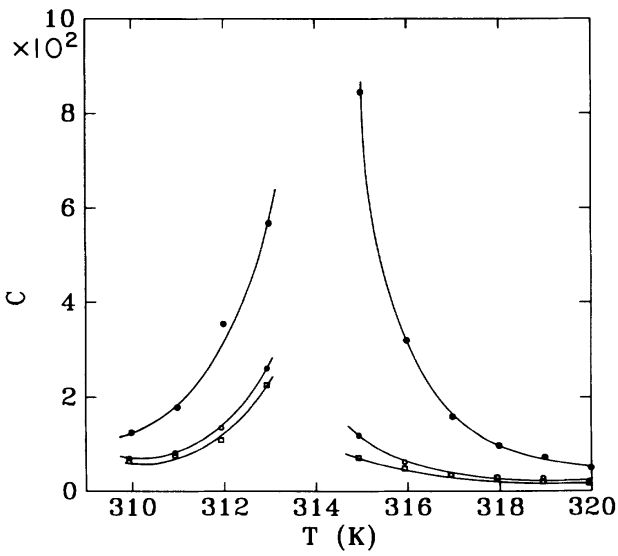

FIG. 12. Specific heat in the thermodynamic limit for temperatures outside the transition region shown for $\gamma_{\mathrm{mis}}=$ $0,4,5 \times 10^{-16} \mathrm{erg} / \AA$ (from top to bottom). The specific heat is in units of $k_{B}$. 


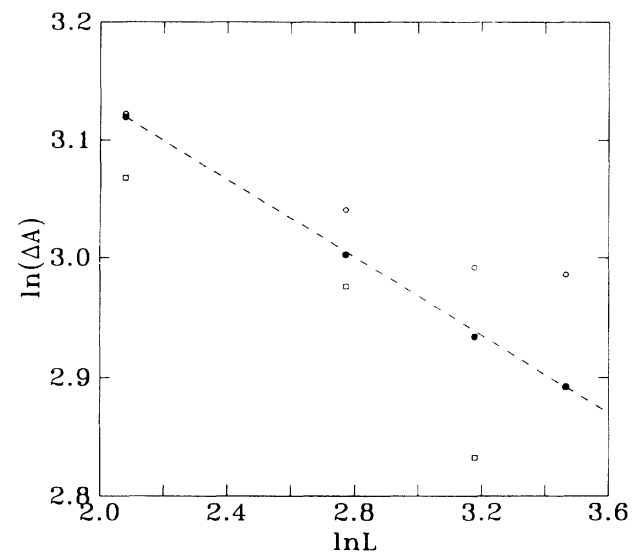

FIG. 13. $\Delta A$ as a function of $L$ for $\gamma_{\text {mis }}=3,4,5 \times$ $10^{-16} \mathrm{erg} / \AA$ (from bottom to top). The result for $\gamma_{\mathrm{mis}}=$ $4 \times 10^{-16}(\bullet)$ shows that $\Delta A \sim L^{-a}$, where $a=0.16$. This scaling exponent is difficult to interpret since an order parameter for the PGC model has not been rigorously defined.

tion away from criticality into a region of clear first-order transitions. The effect of suppressing the fluctuations around the transition region is illustrated in Fig. 12 in the case of the specific heat for the model in Eqs. (1)-(3). The heat content in the specific heat $(\sim 8-9 \mathrm{kcal} / \mathrm{mol})$ as obtained by integration over a narrow temperature range around the transition depends only weakly on the value of $\gamma_{\text {mis }}$ in the first-order regime. However, when the transition becomes of first order, some of the heat is derived from the $\delta$-function singularity of the specific heat. The stronger the first-order transition becomes, the larger the part of the heat involved in the transition region that is due to the latent heat of the transition.

Experimentally, it is virtually impossible to discern between a weakly first-order transition and a pseudotransition that is dominated by very strong fluctuations. A further complication arises because the actual experimental preparation method used to produce aqueous suspensions of the lipid bilayers (vesicles) has a strong influence on the specific-heat profile [2]. Specifically, the specific heat of a multilamellar dispersion appears to be much sharper and less dominated by fluctuations, i.e., of stronger first order, than that of a preparation of unilamellar vesicles. Added to this complication is the problem of aquiring thermodynamic equilibrium in the system. Moreover, the multilamellar dispersion is not a well-defined system. Unilamellar vesicles may also become less well defined when taken through the transition, due to breaking and resealing of the closed membranes. Biltonen [2] has presented experimental evidence based on calorimetry suggesting that the main phase transition of DPPC multibilayer systems is a first-order phase transition with a small transition enthalpy, but that the wings of the specific heat that are very close to the transition are dominated by fluctuations and carry a major contribution to the heat of the transition. This is in agreement with the conclusions of Ipsen, Jørgensen, and Mouritsen [6] and implies that the main phase transition for these systems may be above but close to the critical point found in our calculations. For this reason we have made some preliminary calculations close to the critical point, using our model. Figure 13 gives a log-log plot of the change in area per acyl chain $\Delta A$ at the transition as a function of system size for several values of $\gamma_{\text {mis }}$. This figure shows that $\Delta A$ scales algebraically with system size at the critical value, $\gamma_{\text {mis }}=4 \times 10^{-16} \mathrm{erg} / \AA$, as expected for a continuous transition. These data, taken together with our analysis of the specific-heat behavior near the critical point, Figs. 10 and 12 , should prove useful to further our understanding of the nature of the main lipid bilayer phase transition as more accurate and reliable experimental data on more well-defined sample preparations become available.

\section{ACKNOWLEDGMENTS}

This work was supported by FCAR du Quebec under a centre and team grant, by NSERC of Canada, and by the Danish Natural Science Research Council, under Grant No. 11-7785. The stay of M.J.Z. and Z.Z. in Denmark was supported by the Danish Research Academy under Grant No. S910015. Eugenia Corvera is thanked for useful discussions. The authors also wish to thank John Hjort Ipsen for discussions and for providing results of unpublished work. O.G.M. received support from the Canadian Institute of Advanced Research.
* On leave from the Centre for the Physics of Materials, Department of Physics, McGill University, Montréal, Québec, Canada H3A 2T8.

[1] G. Cevc and D. Marsh, Phospholipid Bilayers. Physical Principles and Models (Wiley-Interscience, New York, 1987).

[2] R.L. Biltonen, J. Chem. Thermodyn. 22, 1 (1990).

[3] J. Lee and J.M. Kosterlitz, Phys. Rev. Lett. 65, 137 (1990).

[4] D.A. Pink, T.J. Green, and D. Chapman, Biochemistry 20, 6692 (1981).

[5] O.G. Mouritsen, in Molecular Description of Biological Membrane Components by Computer Aided Conforma- tional Analysis, edited by R. Brasseur (CRC, Boca Raton, FL, 1990), Vol. 1, p. 3.

[6] J.H. Ipsen, K. Jørgensen, and O.G. Mouritsen, Biophys. J. 58, 1099 (1990).

[7] E. Corvera, M. Laradji, and M. J. Zuckermann (unpublished).

[8] J.H. Ipsen (unpublished data).

[9] O.G. Mouritsen and M. Bloom, Biophys. J. 46, 141 (1984).

[10] K. Binder, Phys. Rev. Lett. 41, 693 (1981).

[11] A.M. Ferrenberg and R.H. Swendsen, Phys. Rev. Lett. 61, 2635 (1988).

[12] J. Lee and J.M. Kosterlitz, Phys. Rev. B 43, 3265 (1991). 
[13] J. Seelig and A. Seelig, Q. Rev. Biophys. 13, 18 (1980).

[14] M.M. Sperotto and O.G. Mouritsen, Biophys. J. 59, 261 (1991).

[15] M.S.S. Challa, D.P. Landau, and K. Binder, Phys. Rev. B 34, 1841 (1986).
[16] Z. Zhang, O. G. Mouritsen, and M. J. Zuckermann (unpublished).

[17] For a list of references to pseudocritical phenomena in lipid bilayers, see Ref. [5] and O. G. Mouritsen and M. J. Zuckermann, Eur. Biophys. J. 12, 75 (1985). 